\title{
Stable 3D Head Direction Signals in the Primary Visual Cortex
}

\author{
Grigori Guitchounts ${ }^{1,2,3,5}$, William Lotter ${ }^{1}$, Joel Dapello ${ }^{4}$, David Cox ${ }^{1,2,4}$ \\ ${ }^{1}$ Center for Brain Science, Harvard University, Cambridge, Massachusetts 02138, USA \\ ${ }^{2}$ Department of Molecular and Cellular Biology, Harvard University, Cambridge, Massachusetts, 02138, USA \\ ${ }^{3}$ Program in Neuroscience, Harvard University, Cambridge, Massachusetts 02138, USA \\ 4 School of Engineering and Applied Sciences, Harvard University, Cambridge, Massachusetts 02139, USA \\ ${ }^{5}$ Correspondence: guitchounts@fas.harvard.edu
}

\begin{abstract}
The mammalian brain's navigation system is informed in large part by visual signals. While the primary visual cortex (V1) is extensively interconnected with brain areas involved in computing head direction (HD) information, it is unknown to what extent navigation information is available in the population activity of visual cortex. To test whether information about head direction information is available in visual cortex, we recorded neuronal activity in V1 of freely behaving rats. We show that significant information about yaw, roll, and pitch of the head can be linearly decoded from V1 either in the presence or absence of visual cues. Individual V1 neurons were tuned to head direction, with a quarter of the neurons tuned to conjunctions of angles in all three planes. These results demonstrate the presence of a critical navigational signal in a primary cortical sensory area and support predictive coding theories of brain function.
\end{abstract}

Keywords: Navigation, Head Direction, Visual Cortex, Predictive Coding, Movement, Naturalistic Behavior

\section{Introduction}

Navigation is a core cognitive process that is critical for survival. The mammalian brain's navigation systems contain specialized cells whose dynamics reflect a sense of location-via place, grid, and border cells-and heading direction ${ }^{1,2}$. Head direction (HD) cells are found in a number of species ${ }^{3-9}$ and brain areas; in rodents, these include the thalamus, hippocampal formation, and the neocortex ${ }^{10-16}$. HD cells rely on vestibular signals relayed via brainstem nuclei ${ }^{10}$, as well as visual signals sent from visual areas to the retrosplenial cortex and subiculum, and superior colliculus inputs to the laterodorsal nucleus of the thalamus ${ }^{11}$. While the influence of visual cues on navigational variables has been studied extensively, it remains largely unknown what effect, if any, navigational variables have on visual representation.

While visual cortical areas have traditionally been thought of as processing centers that transform and analyze incoming sensory information ${ }^{17}$, there is a growing awareness that feedback and modulatory inputs from other modalities have profound effects on visual cortex dynamics ${ }^{18-21}$. Neurons in primary visual cortex (V1) have been shown to signal running speed ${ }^{22}$, increase the gain on visual stimulus during locomotion ${ }^{18}$, and even to signal the direction of movement of the head in the presence or absence of visual input ${ }^{23}$.

Importantly, V1 neurons have also been shown to encode a subjective sense of position in physical space, reminiscent of hippocampal place cells ${ }^{24-27}$. While the functional purpose and circuit mechanisms for place representation in V1 are not clear, some have suggested that these signals represent the brain's internal model of the world, as part of a predictive coding framework ${ }^{28}$. Nevertheless, an outstanding question is what functional purpose, if any, such navigational signals serve for sensory processing. 
A major source of non-visual input to V1 comes from the retrosplenial cortex (RSC), a multimodal area known to contain HD cells ${ }^{19,29-31}$. RSC inputs to V1 have been shown to reflect movement ${ }^{20}$, but the full functional consequences of these inputs on V1 are not well understood, and the extent to which V1 activity represents head direction is an open question. To test whether V1 dynamics reflect HD, we recorded neuronal activity in V1 of freely behaving rats in an open arena home-cage. Half of the sessions were recorded in the dark in order to assess non-visual HD representations in V1. We found that V1 dynamics encoded yaw, roll, and pitch of the head, which constitute the 3D components of HD. These features could be decoded well above chance levels with a simple linear decoder, in either the dark or the light. Many individual V1 neurons were tuned to 3D HD, with about a quarter of cells tuned to conjunctions of preferred angles in all three planes. Altogether, these results indicate that the HD signal extends to a primary sensory cortical area, and suggest that navigational signals are more widespread than previously known.

\section{Results}

\section{Decoding Head Direction}

To investigate the possibility that V1 encodes HD information, we recorded neuronal activity using tetrode arrays targeting layer $2 / 3$ of rat V1 while the animals behaved freely in a home-cage arena (Figs. 1a-d, S1a). Movements were captured using a head-mounted inertial measurement unit (IMU). We focused our analysis on the Euler angles - yaw (a.k.a. azimuth), roll, and pitch - which allowed analysis of 3D direction of the head in allocentric room coordinates. Recordings were performed continuously, 24/7, with pseudo-randomized dark or light epochs that overrode the animals' natural dark-light cycles to control for possible circadian effects. The continuous recordings were split into $\sim 2$-hour dark or light sessions for analysis purposes, which allowed us to examine HD signals in the absence or presence of visual inputs, respectively. Precautions were taken to ensure light levels in the behavioral box in the dark were lower than what is likely required to orient in space using visual landmarks (see Materials and Methods), signifying that any HD signals in V1 in the dark correspond to an estimate based on nonvisual signals (e.g. vestibular, somatosensory, auditory, or olfactory).

Animals explored a uniform range of the yaw component of 3D HD and concentrated the roll and pitch components of 3D HD around zero and 50 degrees below the horizon, respectively, as previously reported (Fig. 1e) ${ }^{32,33}$. While the animals tended to move somewhat more in the dark than in the light (Fig. S1b), there were no systematic differences in behavioral coverage between dark and light sessions (Fig. 1e).

To address whether V1 neural dynamics reflect 3D HD, we built a linear regression model that took as inputs the multiunit activity (MUA) firing rates from the 16 tetrodes, and predicted the HD in the three Euler angles separately (Figs. S1c, S2a-c) (see Materials and Methods). The models performed well above chance, both in the dark and in the light (Fig. 1f-h). Decoding performance was highest for the roll and pitch components and did not differ significantly between the light and dark conditions for any of the planes (Fig. 1h). Decoding performance increased as a function of MUA window size, and was generally highest when the lag between neuronal activity and HD was close to zero milliseconds (Fig. 1i,j).

In previous work, we reported that V1 responses to head orienting movements (HOMs) depend on secondary motor cortex (M2) ${ }^{23}$. To address the possibility that V1 HD encoding also depend on M2, we fit and tested the linear regression model to sessions recorded in M2-lesioned rats. These models performed well in the dark and the light, for yaw, roll, and pitch, albeit with reduced performance for pitch relative to non-lesioned animals (Fig. S2d). Thus, neuronal dynamics in V1 encode the three angular components of HD in freely behaving rats, in a manner that likely does not depend on M2. 
bioRxiv preprint doi: https://doi.org/10.1101/2020.09.04.283762; this version posted September 5, 2020. The copyright holder for this preprint (which was not certified by peer review) is the author/funder, who has granted bioRxiv a license to display the preprint in perpetuity. It is made available under aCC-BY-NC-ND 4.0 International license.

A
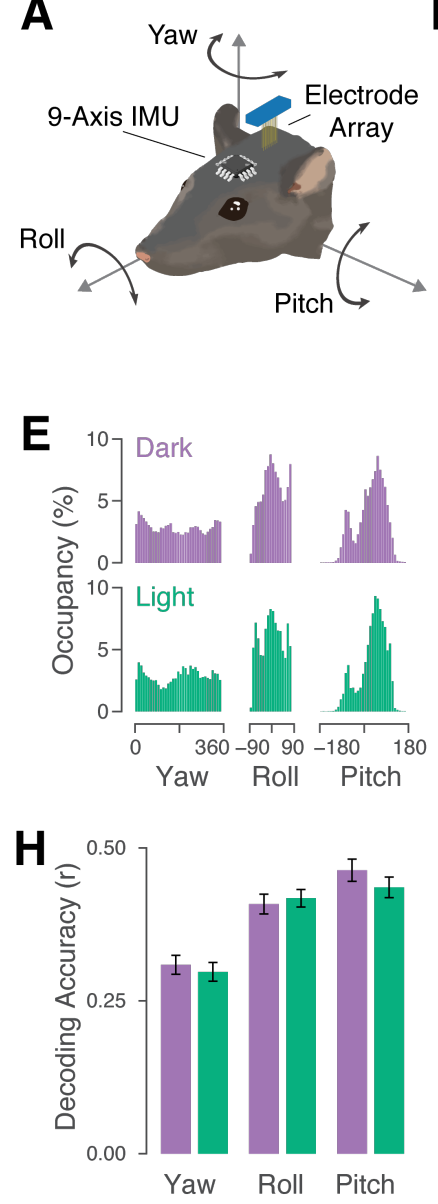

B 24/7 recordings split into 2-hour Dark or Light sessions

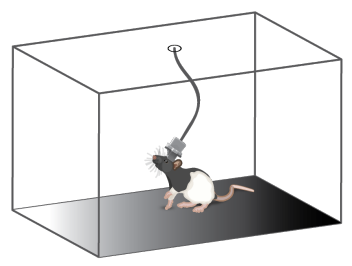

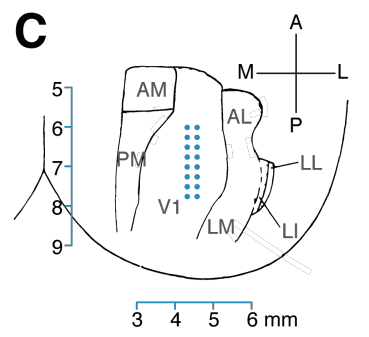

D

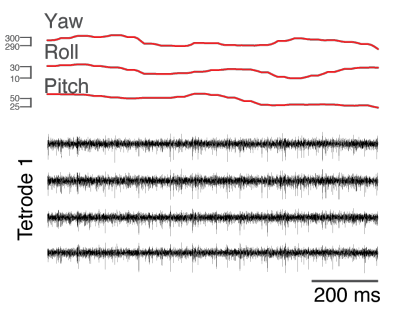

$\mathbf{F}$

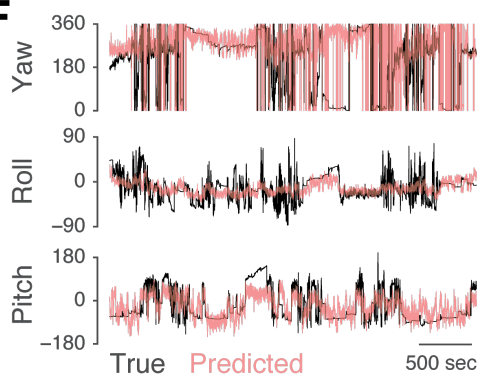

I

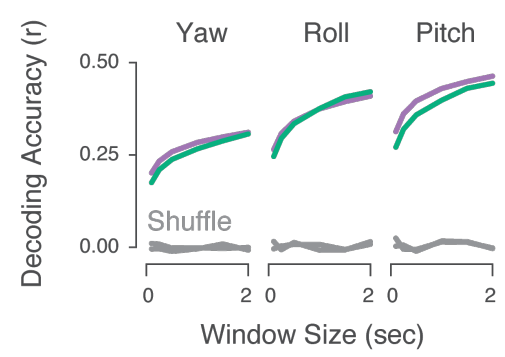

G

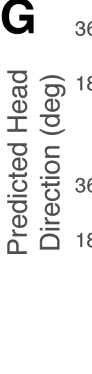

Yaw
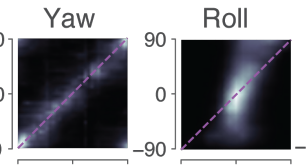

Pitch

J

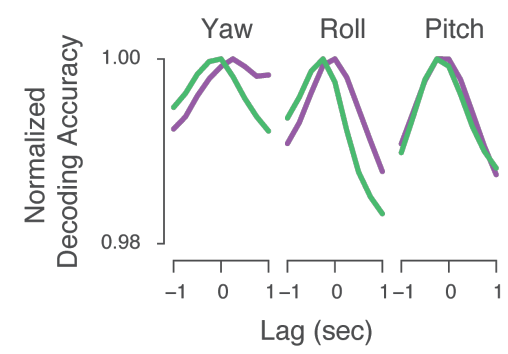

Figure 1: Primary Visual Cortex Encodes 3D Head Direction in Freely Moving Rats. (a) Head direction (HD) angles were measured in 3D using a head-mounted sensor while neural activity in V1 was measured using chronically-implanted electrode arrays. (b) During the 24/7 recordings, rats were free to explore in their home cage either in the light or in the dark, with $\sim 12$ hours per lighting epoch. Each epoch was then split into 2-hour sessions for analysis. (c) Tetrode were arrayed in $8 \mathrm{x} 2$ grids and implanted in V1 along the anterior-posterior axis. (d) Example one-second trace of components of 3D HD (red) and spiking activity from one of the 16 tetrodes (black). (e) Behavioral coverage of the HD angles in dark (purple) and light (green) across rats and sessions. (f) Example traces from one decoding session showing true (black) and predicted (red) HD angles in the dark. Decoding was performed using linear ridge regression with multiunit activity (MUA) firing rates as inputs. (g) Mean bivariate histograms of true and predicted HD angles across rats and sessions in the dark (top row) and light (bottom row). Diagonal lines indicate ideal decoding performance. (h) Summary of decoding model performance measured using the circular correlation coefficient for yaw models and Pearson's correlation coefficient for the roll and pitch models. Yaw, dark: $r_{\text {circ }}=0.31 \pm 0.0154$, light $r_{\text {circ }}=0.30 \pm 0.0154$ (mean $\pm \mathrm{SEM}$ ); Roll, dark $r=0.41 \pm 0.0162$, light $r=0.42 \pm 0.0144$; Pitch, dark $r=0.46 \pm 0.0182$, light $r=0.44 \pm 0.0167$ (mean \pm s.e.m.). (i) Decoding performance as a function of MUA window size for dark or light sessions, or those in which the HD traces were randomly permuted before model fitting (grey). Window lag was 0 seconds for these models. (j) Decoding performance as a function of window lag while window size was held constant at 500 ms. Negative lags represent cases where neural activity occurs before a given HD point; positive lags indicate cases where neural activity occurs after a given HD point. 
bioRxiv preprint doi: https://doi.org/10.1101/2020.09.04.283762; this version posted September 5, 2020. The copyright holder for this

\section{V1 Single Units are Tuned to Individual Components of 3D HD}

To define the cellular basis for the 3D HD signal in V1, we spike-sorted well-isolated units (Fig 2a), which were then classified into regular-spiking putative excitatory units (RSUs) and fast-spiking putative inhibitory units (FSUs) based on waveform shape (Fig S3). V1 neurons showed clear tuning to individual components of 3D HD (Fig. 2b-d), with mean firing rates peaking at particular directions along any given HD axis for many cells. Neurons recorded simultaneously showed a wide degree of directional preference, spanning the extents of each plane (Fig. 3a). Across all recorded cells that showed directional tuning, preferred directions were widely distributed for yaw and pitch, but concentrated for roll (Fig. 3b).

The majority of V1 single units were tuned to yaw, while fewer were tuned to roll or pitch. Nevertheless, substantial fractions of RSUs and FSUs were tuned conjunctively to angles in two planes, with $\sim 20-25 \%$ tuned to angles in all three planes (Fig. 3c,d). These results suggest that at least some neurons in V1 are partially tuned to particular directions in $3 \mathrm{D}$ space.

A Sorting Spikes into Single Units
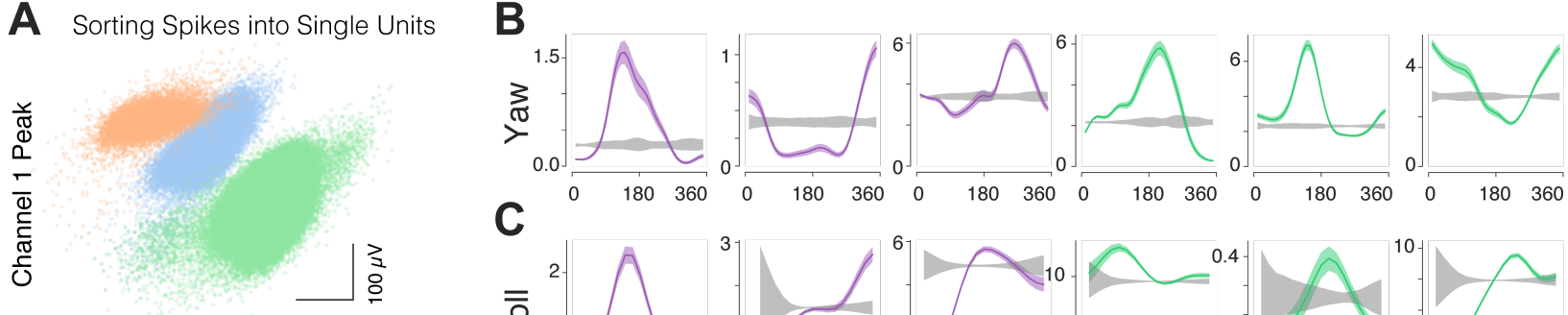

Channel 2 Peak
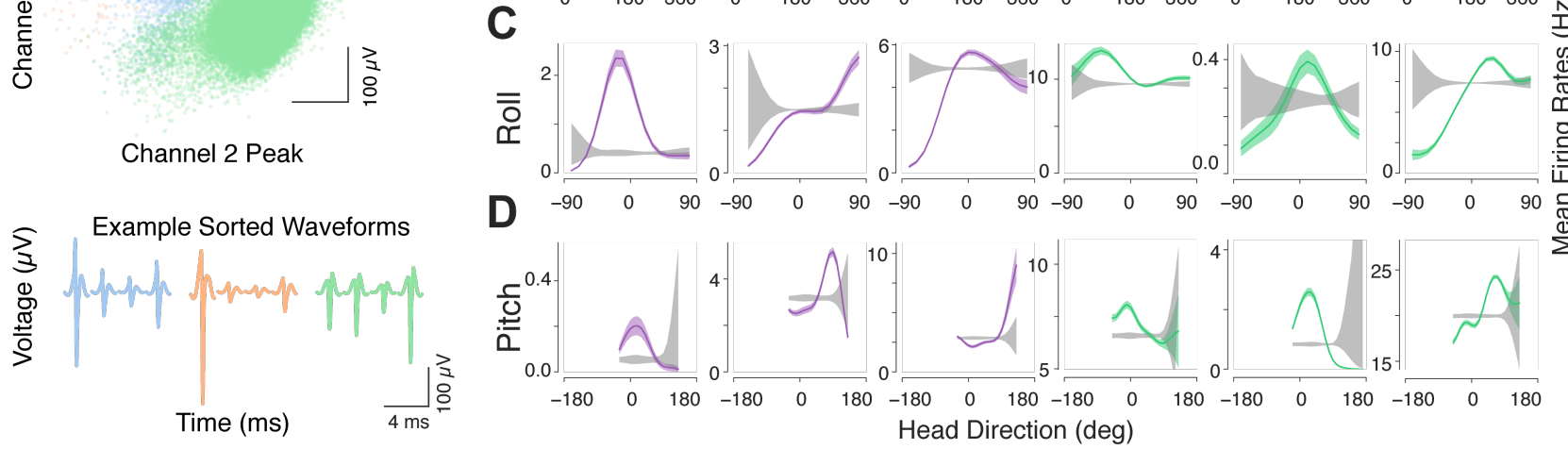

Figure 2: V1 Single Units are Tuned to Individual Components of 3D HD. (a) Spikes extracted from tetrodes were sorted into individual units based on waveform properties using Mountainsort ${ }^{34}$. Top: example peak spike amplitudes from two channels on one tetrode. Colors indicate individual clusters from the sorting algorithm. Bottom: mean waveforms from each of the clusters, showing waveforms from each channel within the example tetrode. (b-d) Examples of 1D tuning curves from 18 single units in V1 recorded in dark (purple) or light (green), with firing rates (mean \pm SEM) plotted as a function of head direction along the yaw (b), roll (c), and pitch (d) components. Grey shading: 95 CI of shuffle distributions.

\section{Head Direction Tuning Stability}

To be useful for navigation, ideally HD representations should be stable over time and invariant to environmental conditions. To examine the stability of the 3D HD signal in V1, we analyzed the correlation structure of weights from the MUA linear regression models used to decode 3D HD over time. Each session's weights (200 time-points per tetrode $\times 16$ tetrodes) were correlated to weights from sessions from the same rat separated by 0-24, 24-48, 48-96, or 96-192 hours, and compared to correlations of weights taken from different rats (Fig. 4a). We reasoned that weight correlations from across rats should serve as a control measure, since there is no reason to expect tetrodes from different brains to be weighted similarly. We found that within-rat weight correlations were higher than across-rat correlations for all models, and especially for the roll and pitch, in both dark and light. These results indicate that the 3D HD representation exhibits stability for up to one week. 

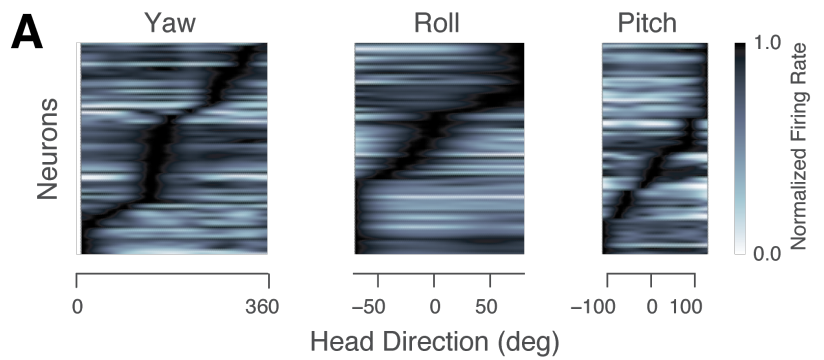

B
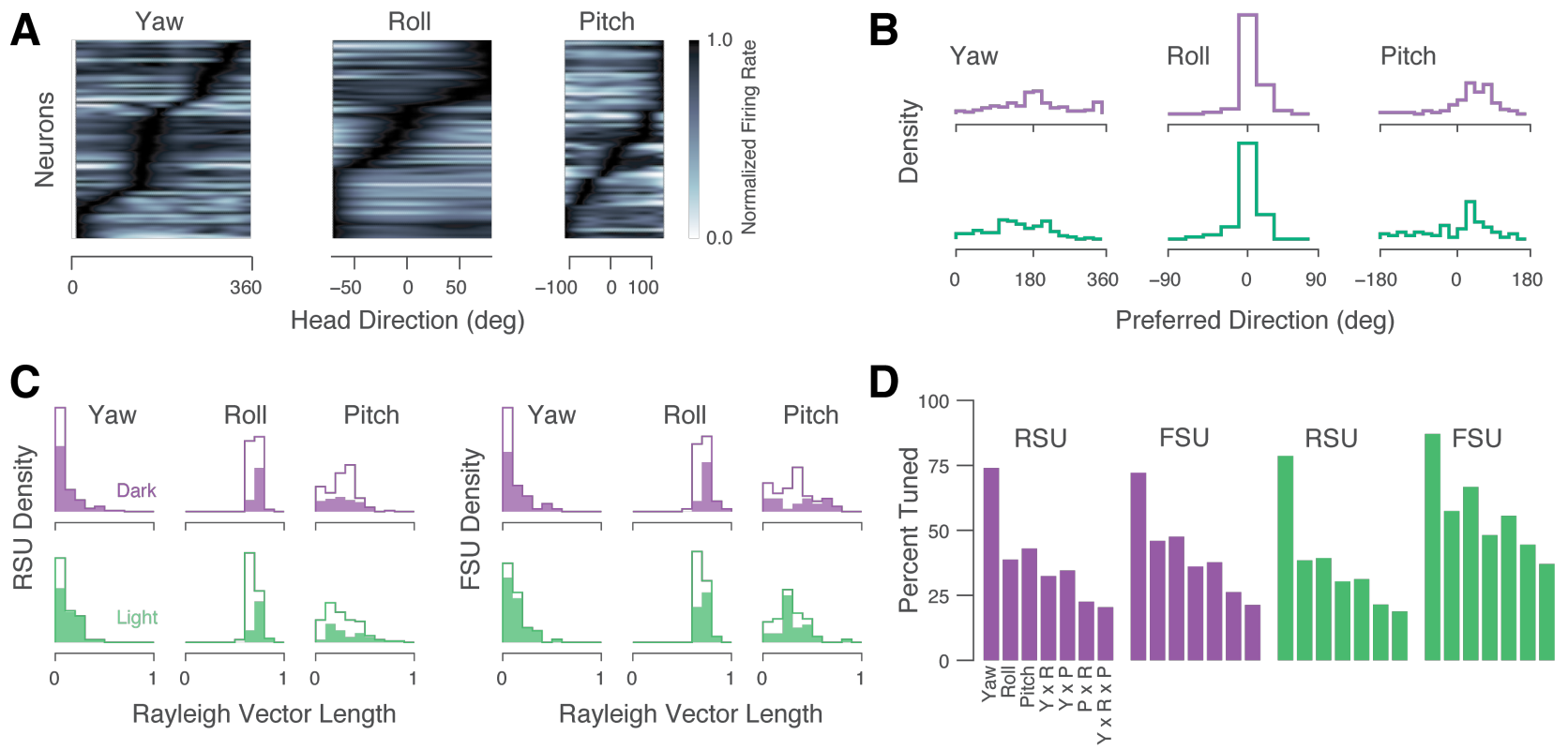

Figure 3: Directional Coverage of Tuned Cells. (a) 3D HD activity of 71 simultaneously-recorded V1 neurons in the dark. Neurons sorted by the time of the peak-normalized firing rates. (b) Preferred directions of all neurons with significant tuning. (c) Histograms of Rayleigh vector lengths for RSUs (left) and FSUs (right) in dark (purple, top row) and light (green, bottom row). Unfilled lines indicate all cells; filled area represents significantly-tuned cells. Note: random vector length on the -90-90 degree interval (roll) is 0.6 . (d) Percentages of tuned neurons to individual components of 3D HD (yaw, roll, pitch) or combinations of two or three angles.

To address how visual inputs may impact HD tuning of individual neurons in V1, we tracked single units between the dark and light conditions for a subset of experiments. While mean firing rates were different between the dark and light conditions (data not shown), z-scored tuning curves of many neurons were similar across the two lighting conditions (Fig. $4 \mathrm{~b}$ ), and $\sim 60 \%$ of the cells were tuned to HD in both conditions. Examining the differences in preferred direction of significantly-tuned neurons revealed that most neurons had low directional discrepancy between light and dark, although the drift was higher for yaw, as has previously been reported for recordings performed in the dark in subcortical head direction circuits ${ }^{14,35}$ (Fig. 4c). Together, these results indicate that while the directional tuning may drift somewhat in the absence of visual cues, many individual V1 neurons displayed tuning stability across light and dark conditions, and the multiunit signal that formed the basis for the linear decoding of 3D HD was stable for at least one week.

\section{Discussion}

Using chronic electrophysiological recordings in freely behaving rats, we showed that primary visual cortex encodes three-dimensional components of head direction, both in the presence and absence of visual cues. This signal was stable both across time and lighting conditions.

While several previous studies have failed to find HD signals that extend beyond yaw angle (azimuth) in the rodent limbic system ${ }^{36,37}$, recent work has validated the idea that navigational variables represent threedimensional space ${ }^{38}$. 3D HD cells were found in the presubiculum of crawling or flying bats ${ }^{39}$, while $3 \mathrm{D}$ place cells were found in both bat and rat hippocampus ${ }^{40,41}$. It is not entirely clear why some studies have failed to 
A

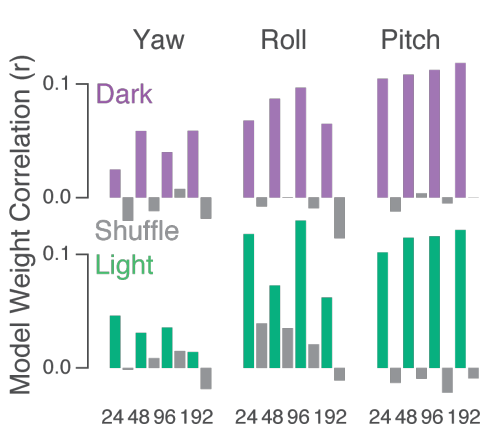

Time Between Sessions (hours)
B

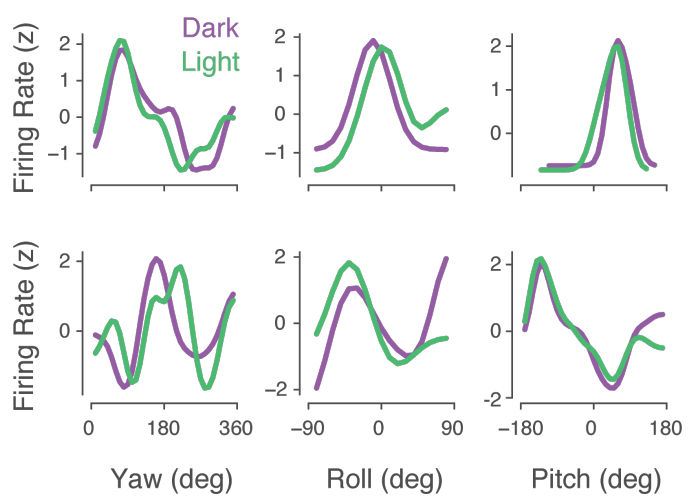

C

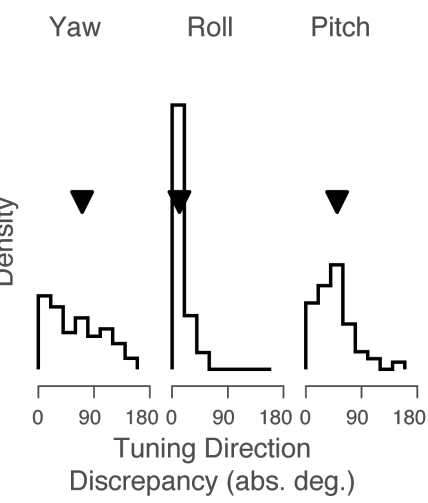

Figure 4: Encoding of Head Direction by V1 Neurons is Stable. (a) Correlations of MUA ridge regression model weights among session pairs separated by 0-24, 24-48, 48-96, or 96-192 hours. Purple (dark) and green (light) indicate session pairs for one given rat (i.e. within-rat comparisons); grey bars indicate correlations among session pairs from different rats (i.e. across-rat comparisons). Sessions with above-median decoding performance were used for the within-rat comparison. (b) Example 1D tuning curves of neurons recorded simultaneously in dark (purple) and light (green) showing stability of tuning between the two conditions. (c) Histogram of tuning direction discrepancy (absolute circular difference) between dark and light. Carets indicate means. Yaw: $69.04 \pm 6.69$ degrees; roll: $13.85 \pm 1.70$; pitch: $54.47 \pm 5.80$ (mean \pm SEM).

find 3D HD cells in the rodent limbic system, but one possible explanation is methodological: rats in the present study were free to move as they wished and head movements were measured using a head-mounted IMU, while in some previous studies animals were required to crawl upside down along a ceiling ${ }^{14}$ or restrained and moved passively $^{37}$. Further work into naturalistic behavior in 3D space may resolve this issue.

While the head direction information that we have decoded could reasonably be the product of visual processing in the presence of light, the fact that these signals are also present (and stable) in the dark, points to an exogenous source of this information. Retrosplenial cortex (RSC) is one plausible candidate for this source, as it processes vestibular information and sends extensive projections to V1. What purpose, if any, might head direction representation in a primary visual area serve? One possibility is that head-direction representations in visual cortex play a role in reconciling visually-driven and vestibular contributions to an overall representation of head direction. Elegant theoretical work has posited a ring attractor as a possible computational substrate for estimating head direction in the brain, with input from multiple modalities ${ }^{42}$; a literal ring of neurons implementing a ring attractor that computes head direction has recently been described in the brains of fruit flies $^{3-6}$. While our results don't definitively speak to the presence of such a system in the brain of the rat, the presence of stable, persistent representations of heading from multiple modalities could play a role in such a system if it exists.

Another, non-mutually-exclusive, possible explanation for HD signals in V1 is that these are used by visual circuits to aid in predicting future visual features. This paradigm proposes that a core function of the visual cortical hierarchy is to make predictions about future states ${ }^{28,43-45}$. The finding here that primary visual cortex contains information about head direction suggests an intriguing possibility that such navigational signals may be used by the visual cortex to improve predictions about future visual features, as the animal moves through the environment. Future work may test this idea experimentally in vivo, for example by isolating and manipulating V1-projecting axons containing HD information in the context of a behavioral task.

In previous work, we demonstrated that rat V1 responds to orienting movements of the head in a directionand light-dependent manner ${ }^{23}$. We found also that V1 responses to such movements were severely reduced 
following lesions to secondary motor cortex (M2), even though the movements themselves were relatively unaffected. Applying the HD decoding models to M2-lesioned animals showed that V1 in these animals still encoded 3D HD (Fig. S2d), suggesting that HD signals in V1 originate in a different region.

While it is broadly accepted that vision plays a significant role in shaping the brain's sense of space and heading direction, little work has been done to ask how the computation of navigational variables impacts dynamics in sensory areas. We have taken a first step toward elucidating the interplay of visual sensory signals and those that encode head direction. Future work will investigate exactly where these signals originate, and how they impact sensory perception and behavior. While the RSC, which contains cortical HD cells, projects heavily to $\mathrm{V}^{19}$, it is also possible that $\mathrm{V} 1$ inherits this information indirectly from parahippocampal or thalamic regions. More experiments will be needed to establish the source of non-visual head direction information in V1.

This work adds to a growing awareness that non-visual signals play a critical role in shaping visual cortical population dynamics, and that the computational role of visual cortex may extend beyond just processing visual inputs. Understanding how the visual cortex interacts with other multimodal systems of the brain, such as a navigation, holds the promise to deepen our understanding of cortical computation in general.

\section{Materials and Methods}

\section{Animals}

The care and experimental manipulation of all animals were reviewed and approved by the Harvard Institutional Animal Care and Use Committee. Experimental subjects were female Long Evans rats 3 months or older, weighing 300-500 g ( $n=9$, Charles River, Strain Code: 006).

\section{Surgery}

Rats were implanted with 16-tetrode electrode arrays targeting L2/3 of V1, as described previously ${ }^{23}$. Animals were anesthetized with $2 \%$ isoflurane and placed into a stereotaxic apparatus (Knopf Instruments). Care was taken to clean the scalp with Povidone-iodine swabsticks (Professional Disposables International, \#S41125) and isopropyl alcohol (Dynarex \#1204) before removing the scalp and cleaning the skull surface with hydrogen peroxide (Swan) and a mixture of citric acid (10\%) and ferric chloride (3\%) (Parkell \#S393). Three to four skull screws (Fine Science Tools, \#19010-00) were screwed into the skull to anchor the implant. A 0.003 " stainless steel (A-M Systems, \#794700) ground wire was inserted $\sim 2 \mathrm{~mm}$ tangential to the brain over the cerebellum.

Tetrodes were arrayed in $8 \times 2$ grids with $\sim 250$-micron spacing, and were implanted in V1 with the long axis spread along the AP (ranging 6-8 $\mathrm{mm}$ posterior to bregma, $4.5 \mathrm{~mm} \mathrm{ML}$, targeting layer $2 / 3$ at $0.6 \mathrm{~mm} \mathrm{DV}$ ). The dura was glued (Loctite) to the edges of the craniotomy to minimize movement of the brain relative to the electrodes. After electrodes were inserted into the brain, the craniotomy was sealed with Puralube vet ointment (Dechra) and the electrodes were glued down with Metabond (Parkell). Post-operative care included twice-daily injections of buprenex $(0.05 \mathrm{mg} / \mathrm{kg}$ Intraperitoneal (IP)) and dexamethasone $(0.5 \mathrm{mg} / \mathrm{kg}$ IP) for three days.

\section{Behavior}

Spontaneous behavior in rats living in a 15x24" home cage was recorded under two conditions: dark, in which the lights in the box and room were turned off, and light, in which the box was illuminated. Recordings were carried out $24 / 7$ and split into 2 -hour sessions in dark or light on a pseudo-random light cycle, as previously described $^{23}$.

The behavior box was constructed from aluminum extrusions and black extruded acrylic (McMaster). The floor was covered in bedding and the arena contained a cup with food, a water bottle and toys. The walls were 
lined with strips of white tape at different orientations to provide visual features in the light condition, and the box was outfitted with white LED strips (Triangle Bulbs Cool White LED Waterproof Flexible Strip Light, T93007-1, Amazon) to provide illumination.

For recordings, rats were tethered with a custom 24" cable (Samtec, SFSD-07-30C-H-12.00-DR-NDS, TFM107-02-L-D-WT;McMaster extension spring 9640K123) to a commutator (Logisaf 22mm 300Rpm 24 Circuits Capsule Slip Ring 2A 240V TestEquipment, Amazon). A 9-axis Inertial Measurement Unit (IMU) (BNO055, Adafruit) was used to record movement; the sensor was epoxied to the connector on the cable, in a way that placed it directly above the electrodes and headstage. This not only ensured that the sensor was always in the same position above the animals' heads, but also that it stayed powered after the animals were unplugged, preventing the need to re-calibrate the sensor after each recording. The IMU data were acquired at $100 \mathrm{~Hz}$ using a micro-controller (Arduino) and saved directly to the acquisition computer's disk. To synchronize IMU and electrophysiology data, the Arduino provided a 2-bit pseudo-random pulse code to the TTL inputs on the electrophysiology system.

\section{Electrophysiology}

Tetrodes were fabricated using 12.5-micron nichrome wire (Sandvik-Kanthal) following standard procedures $^{46-48}$, as described previously ${ }^{23}$. Tetrodes were threaded through 42 AWG polyimide guide tubes into $8 \times 2$ grids of 34 AWG tubes (Small Parts) and glued to a single-screw micro-drive. The drive was modified from a

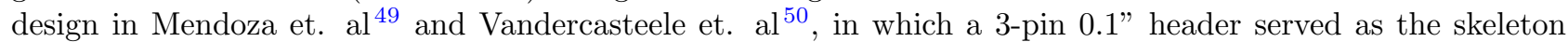
of the drive, with a \#0-80 screw replacing the middle pin, and the header's plastic serving as the shuttle. In the experiments reported here, the tetrodes were not advanced after recording sessions started. The tetrodes were plated with a mixture of gold (Neuralynx) and polyethylene glycol (PEG) as per Ferguson et. al ${ }^{51}$, to an impedance of $\sim 100-250 K \Omega$. The ground and reference wires were bridged and implanted through a craniotomy above the cerebellum.

Electrode signals were acquired at $30 \mathrm{kHz}$ using custom-made Intan-based 64-channel headstages ${ }^{52}$ and OpalKelly FPGAs (XEM6010 with Xilinx Spartan-6 ICs). Spikes were extracted following procedures described in Dhawale et al $(2017)^{52}$. Multiunit firing rates were estimated in non-overlapping 10-ms bins from extracted spikes. Multiunit and single-unit firing rates were Gaussian-filtered and in some cases z-scored. Single-units were sorted using MountainSort ${ }^{34}$ and classified into putative excitatory regular-spiking units (RSUs) or putative inhibitory fast-spiking units (FSUs) based on the trough-to-peak time (width) and full-width at half-max (FWHM) of the unfiltered waveforms (Fig. S3).

\section{Head Direction Decoding}

Head direction in 3D was decoded separately for the yaw, roll, and pitch angles using Ridge linear regression. Because the yaw signal is circular, it was modeled in the complex plane (i.e. angles were decomposed into $\mathrm{x}$ and y components, fitted to two separate models, and recombined into angles during evaluation).

The models took z-scored MUA firing rates as inputs and predicted the angle of a given component of 3D HD. The MUA firing rates from $n=16$ tetrodes were taken in time windows and flattened before fitting, such that each window $\mathrm{x}$ tetrode became a vector of features used to predict a HD angle (Fig. S2).

The models were fit using half of the data in a session, with the session split into 30-second chunks separated by 0.25 -second discarded gaps. The even chunks were used for fitting the linear regression models and the odd ones were used for testing. The Ridge models were implemented using the Scikit Learn Python package ${ }^{53}$, and cross-validated threefold. Each two-hour session was modeled separately $(n=89$ sessions in dark, $n=95$ sessions in light across $n=5$ rats).

To determine optimal window size and lag, we performed a grid search, with window size ranging from 0.10 , $0.25,0.50,1.00,1.50$, to 2.00 seconds, and window lag ranging from -1.00 to 1.00 seconds in steps of $250 \mathrm{~ms}$. Negative lags represent neural activity lagging behind a given HD position (Fig. 1i,j). 


\section{Lesions}

Lesions of M2 were performed as previously described ${ }^{23}$, using excitotoxic injections of ibotenic acid (IA) (Abcam ab120041) delivered using an UMP3 UltraMicroPump (WPI) during two separate procedures. Aliquots of IA were prepared at $1 \%$ concentration and frozen. In the first procedure, IA was injected into four sites in one hemisphere (1.5 mm AP, relative to Bregma and $1.0 \mathrm{~mm} \mathrm{ML}$; 0.5 AP, 0.75 ML; -0.5 AP, $0.75 \mathrm{ML}$; and $-1.5 \mathrm{AP}, 0.75 \mathrm{ML}$, with two injections per site, at 1.6 and $0.8 \mathrm{~mm}$ below the brain surface, $75 \mathrm{nl}$ each) and the animal was allowed to recover for one week, after which the injections were repeated at the same sites in the opposite hemisphere and electrode arrays were implanted in V1.

\section{Measuring Light Levels in Dark}

To assess whether the recording box was sufficiently dark as to prevent the rats from being able to see anything in the dark recordings, we first made sure that it was impossible for a human observer (GG) to see any movement in the recording box after acclimating to the darkened room for 30 minutes. We then attempted to measure photon flux in the box using a photomultiplier tube (PMT) (ET Enterprises \#9111B) after amplifying and filtering the signals (12dB lowpass at 10Hz) using a Stanford Research Systems preamplifier (\#SR570).

Baseline PMT currents in the darkened behavioral box were measured with the PMT covered by tinfoil. After removing the tinfoil, the PMT current registered at $0.2 \mu \mathrm{A}$. This corresponds to $0.2 * 10^{-6}$ Coulombs/s, which is $0.2 * 6.2415 * 10^{12}$ electrons/s. Accounting for the PMT's gain of $7.1 * 10^{6}$, that is $0.2 * 6.2415 * 10^{12} / 7.1 * 10^{6}$ or $1.8 * 10^{5}$ photocathode events/s. Given the PMT's $10 \%$ quantum efficiency (QE) at $500 \mathrm{~nm}$, this corresponds to $1.8 * 10^{6}$ photons/s over the PMT's $22 \mathrm{~mm}$ cross-sectional area, or 80,000 photons $/ \mathrm{mm} / \mathrm{s}$. Assuming $2.27 \mu m^{2}$ rod cross section ${ }^{54}, 0.4$ specific absorption, and QE of $0.34^{55}$, that is $80000 * 2.27 * 10^{-6}$ or 0.18 incident photons/rod/s and finally $0.025 \mathrm{R} * / \mathrm{rod} / \mathrm{s}$. Based on retinal ganglion cell activity measured in Soucy et al. ${ }^{56}$, $0.025 \mathrm{R}^{*} / \mathrm{rod} / \mathrm{s}$ would correspond, roughly, to retinal ganglion cells firing at $6.7 \%$ of their peak firing rates measured at light levels corresponding to $100 \mathrm{R}^{*} / \mathrm{rod} / \mathrm{sec}$.

\section{Statistics}

All statistical comparisons were done using non-parametric tests (e.g. Mann-Whitney U or Wilcoxon) unless specified otherwise. A significance level of alpha $=0.01$ was used throughout, unless otherwise noted. Bonferroni correction was applied where appropriate.

\section{Acknowledgements}

We would like to thank Jeffrey Markowitz, Bence Ölveczky, and Sandeep Robert Datta, for advice and helpful discussions. Edward Soucy, Brett Graham, and Joel Greenwood of the CBS Neuroengineering Core were instrumentally helpful in technical advice and on light measurements. GG was supported by the National Science Foundation (NSF) Graduate Research Fellowship Program (GRFP).

\section{Author contributions statement}

GG conceived and performed the electrophysiology and lesion experiments, analyzed the data, and wrote the manuscript. WL and JD provided assistance with modeling. DC provided research funding and space.

\section{Additional information}

Declaration of Interests: The authors declare no competing interests. 
bioRxiv preprint doi: https://doi.org/10.1101/2020.09.04.283762; this version posted September 5, 2020. The copyright holder for this

preprint (which was not certified by peer review) is the author/funder, who has granted bioRxiv a license to display the preprint in perpetuity. It is made available under aCC-BY-NC-ND 4.0 International license.

\section{References}

${ }^{1}$ Bellmund, J. L., Gärdenfors, P., Moser, E. I. \& Doeller, C. F. Navigating cognition: Spatial codes for human thinking. Science 362, eaat6766 (2018)

2 Buzsáki, G. \& Moser, E. I. Memory, navigation and theta rhythm in the hippocampal-entorhinal system. Nature neuroscience 16, 130 (2013).

${ }^{3}$ Kim, S. S., Hermundstad, A. M., Romani, S., Abbott, L. \& Jayaraman, V. Generation of stable heading representations in diverse visual scenes. Nature 576, 126-131 (2019).

${ }^{4}$ Kim, S. S., Rouault, H., Druckmann, S. \& Jayaraman, V. Ring attractor dynamics in the drosophila central brain. Science $\mathbf{3 5 6}$, 849-853 (2017).

${ }^{5}$ Seelig, J. D. \& Jayaraman, V. Neural dynamics for landmark orientation and angular path integration. Nature 521, 186-191 (2015).

${ }^{6}$ Fisher, Y. E., Lu, J., D'Alessandro, I. \& Wilson, R. I. Sensorimotor experience remaps visual input to a heading-direction network. Nature 576, 121-125 (2019).

${ }^{7}$ Nau, M., Schröder, T. N., Bellmund, J. L. \& Doeller, C. F. Hexadirectional coding of visual space in human entorhinal cortex. Nature neuroscience 21, 188-190 (2018).

${ }^{8} \mathrm{Nau}$, M., Julian, J. B. \& Doeller, C. F. How the brain's navigation system shapes our visual experience. Trends in cognitive sciences 22, 810-825 (2018).

${ }^{9}$ Nau, M., Schröder, T. N., Frey, M. \& Doeller, C. F. Behavior-dependent directional tuning in the human visual-navigation network. bioRxiv 765800 (2019).

${ }^{10}$ Clark, B. J. \& Taube, J. S. Vestibular and attractor network basis of the head direction cell signal in subcortical circuits. Frontiers in neural circuits 6, 7 (2012).

${ }^{11}$ Yoder, R. M., Clark, B. J. \& Taube, J. S. Origins of landmark encoding in the brain. Trends in neurosciences 34, 561-571 (2011).

12 Cullen, K. E. \& Taube, J. S. Our sense of direction: progress, controversies and challenges. Nature Neuroscience 20, 1465 (2017).

${ }^{13}$ Yoder, R. M. \& Taube, J. S. The vestibular contribution to the head direction signal and navigation. Frontiers in integrative neuroscience 8, 32 (2014).

14 Taube, J. S. The head direction signal: origins and sensory-motor integration. Annu. Rev. Neurosci. 30, 181-207 (2007).

${ }^{15}$ Angelaki, D. E. \& Laurens, J. The head direction cell network: attractor dynamics, integration within the navigation system, and three-dimensional properties. Current Opinion in Neurobiology 60, 136-144 (2020).

${ }^{16}$ Laurens, J. \& Angelaki, D. E. The brain compass: a perspective on how self-motion updates the head direction cell attractor. Neuron 97, 275-289 (2018).

17 DiCarlo, J. J., Zoccolan, D. \& Rust, N. C. How does the brain solve visual object recognition? Neuron 73, 415-434 (2012).

18 Niell, C. M. \& Stryker, M. P. Modulation of Visual Responses by Behavioral State in Mouse Visual Cortex. Neuron 65, 472-479 (2010).

${ }^{19}$ Leinweber, M., Ward, D. R., Sobczak, J. M., Attinger, A. \& Keller, G. B. A Sensorimotor Circuit in Mouse Cortex for Visual Flow Predictions. Neuron 95, 1420-1432.e5 (2017).

${ }^{20}$ Vélez-Fort, M. et al. A Circuit for Integration of Head- and Visual-Motion Signals in Layer 6 of Mouse Primary Visual Cortex. Neuron 1-25 (2018).

${ }^{21}$ Gilbert, C. D. \& Li, W. Top-down influences on visual processing. Nature Reviews Neuroscience 14, 350-363 (2013).

22 Saleem, A. B., Ayaz, A., Jeffery, K. J., Harris, K. D. \& Carandini, M. Integration of visual motion and locomotion in mouse visual cortex. Nature Neuroscience 16, 1864-1869 (2013).

${ }^{23}$ Guitchounts, G., Masís, J., Wolff, S. B. E. \& Cox, D. Encoding of 3D Head Orienting Movements in the Primary Visual Cortex. Neuron 1-27 (2020).

${ }^{24} \mathrm{Ji}$, D. \& Wilson, M. A. Coordinated memory replay in the visual cortex and hippocampus during sleep. Nature neuroscience 10, 100-107 (2007).

${ }^{25}$ Haggerty, D. C. \& Ji, D. Activities of visual cortical and hippocampal neurons co-fluctuate in freely moving rats during spatial behavior. Elife 4, e08902 (2015).

${ }^{26}$ Fiser, A. et al. Experience-dependent spatial expectations in mouse visual cortex. Nature Neuroscience 19, 1658-1664 (2016).

${ }^{27}$ Saleem, A. B., Diamanti, E. M., Fournier, J., Harris, K. D. \& Carandini, M. Coherent encoding of subjective spatial position in visual cortex and hippocampus. Nature 7, 1-18 (2018).

${ }^{28}$ Keller, G. B. \& Mrsic-Flogel, T. D. Predictive Processing: A Canonical Cortical Computation. Neuron 100, 424-435 (2018).

${ }^{29}$ Chen, L. L., Lin, L.-H., Green, E. J., Barnes, C. A. \& McNaughton, B. L. Head-direction cells in the rat posterior cortex. Experimental brain research 101, 8-23 (1994).

${ }^{30}$ Chen, L. L., Lin, L. H., Barnes, C. A. \& McNaughton, B. L. Head-direction cells in the rat posterior cortex. ii. contributions of visual and ideothetic information to the directional firing. Experimental brain research 101, 24-34 (1994).

${ }^{31} \mathrm{Jacob}$, P.-Y. et al. An independent, landmark-dominated head-direction signal in dysgranular retrosplenial cortex. Nature neuroscience 20, 173 (2017).

${ }^{32}$ Wallace, D. J. et al. Rats maintain an overhead binocular field at the expense of constant fusion. Nature 498, 65 (2013). 
${ }^{33}$ Meister, M. \& Cox, D. Rats maintain a binocular field centered on the horizon. F1000Research 2 (2013).

${ }^{34}$ Chung, J. E. et al. A Fully Automated Approach to Spike Sorting. Neuron 95, 1381-1394.e6 (2017).

35 Goodridge, J. P., Dudchenko, P. A., Worboys, K. A., Golob, E. J. \& Taube, J. S. Cue control and head direction cells. Behavioral neuroscience 112, 749 (1998).

36 Calton, J. L. \& Taube, J. S. Degradation of head direction cell activity during inverted locomotion. Journal of Neuroscience 25, 2420-2428 (2005).

37 Shinder, M. E. \& Taube, J. S. Three-dimensional tuning of head direction cells in rats. Journal of neurophysiology 121, 4-37 (2019).

38 Angelaki, D. E. et al. A gravity-based three-dimensional compass in the mouse brain. Nature communications 11, 1-13 (2020).

39 Finkelstein, A. et al. Three-dimensional head-direction coding in the bat brain. Nature 517, 159-164 (2015).

40 Yartsev, M. M. \& Ulanovsky, N. Representation of three-dimensional space in the hippocampus of flying bats. Science 340, 367-372 (2013).

${ }^{41}$ Grieves, R. M. et al. The place-cell representation of volumetric space in rats. Nature communications 11, 1-13 (2020).

42 Skaggs, W. E., Knierim, J. J., Kudrimoti, H. S. \& McNaughton, B. L. A model of the neural basis of the rat's sense of direction. In Advances in neural information processing systems, 173-180 (1995).

43 Rao, R. P. \& Ballard, D. H. Predictive coding in the visual cortex: a functional interpretation of some extra-classical receptivefield effects. Nature Neuroscience 2, 79-87 (1999).

${ }^{44}$ Clark, A. Whatever next? predictive brains, situated agents, and the future of cognitive science. Behavioral and brain sciences 36, 181-204 (2013)

${ }^{45}$ Friston, K. A theory of cortical responses. Philosophical transactions of the Royal Society B: Biological sciences 360, 815-836 (2005).

46 Siegle, J. H. et al. Open Ephys: an open-source, plugin-based platform for multichannel electrophysiology. Journal of Neural Engineering 14, 045003-14 (2017).

47 Nguyen, D. P. et al. Micro-drive array for chronic in vivo recording: tetrode assembly. JoVE (Journal of Visualized Experiments) e1098 (2009).

${ }^{48}$ Kloosterman, F. et al. Micro-drive array for chronic in vivo recording: drive fabrication. JoVE (Journal of Visualized Experiments) e1094 (2009).

49 Mendoza, G. et al. Recording extracellular neural activity in the behaving monkey using a semi-chronic and high-density electrode system. Journal of Neurophysiology 116, jn.00116.2016-574 (2016).

50 Vandecasteele, M. et al. Large-scale recording of neurons by movable silicon probes in behaving rodents. JoVE (Journal of Visualized Experiments) e3568 (2012).

${ }^{51}$ Ferguson, J. E., Boldt, C. \& Redish, A. D. Creating low-impedance tetrodes by electroplating with additives. Sensors and actuators. A, Physical 156, 388-393 (2009).

52 Dhawale, A. K. et al. Automated long-term recording and analysis of neural activity in behaving animals. eLife 6, 91 (2017).

53 Pedregosa, F. et al. Scikit-learn: Machine learning in python. Journal of Machine Learning Research 12, 2825-2830 (2011).

54 Hagins, W., Penn, R. \& Yoshikami, S. Dark current and photocurrent in retinal rods. Biophysical journal 10, 380-412 (1970).

55 Nymark, S., Heikkinen, H., Haldin, C., Donner, K. \& Koskelainen, A. Light responses and light adaptation in rat retinal rods at different temperatures. The Journal of physiology 567, 923-938 (2005).

56 Soucy, E., Wang, Y., Nirenberg, S., Nathans, J. \& Meister, M. A novel signaling pathway from rod photoreceptors to ganglion cells in mammalian retina. Neuron 21, 481-493 (1998).

\section{Supplementary Figures}




\section{A}
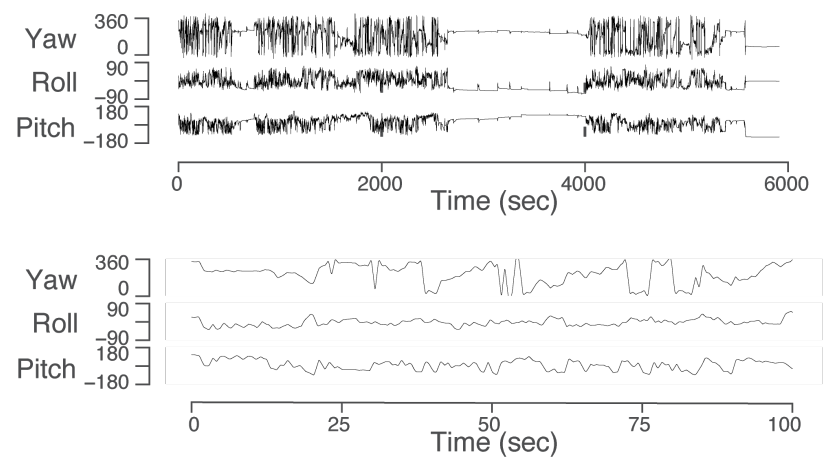

B
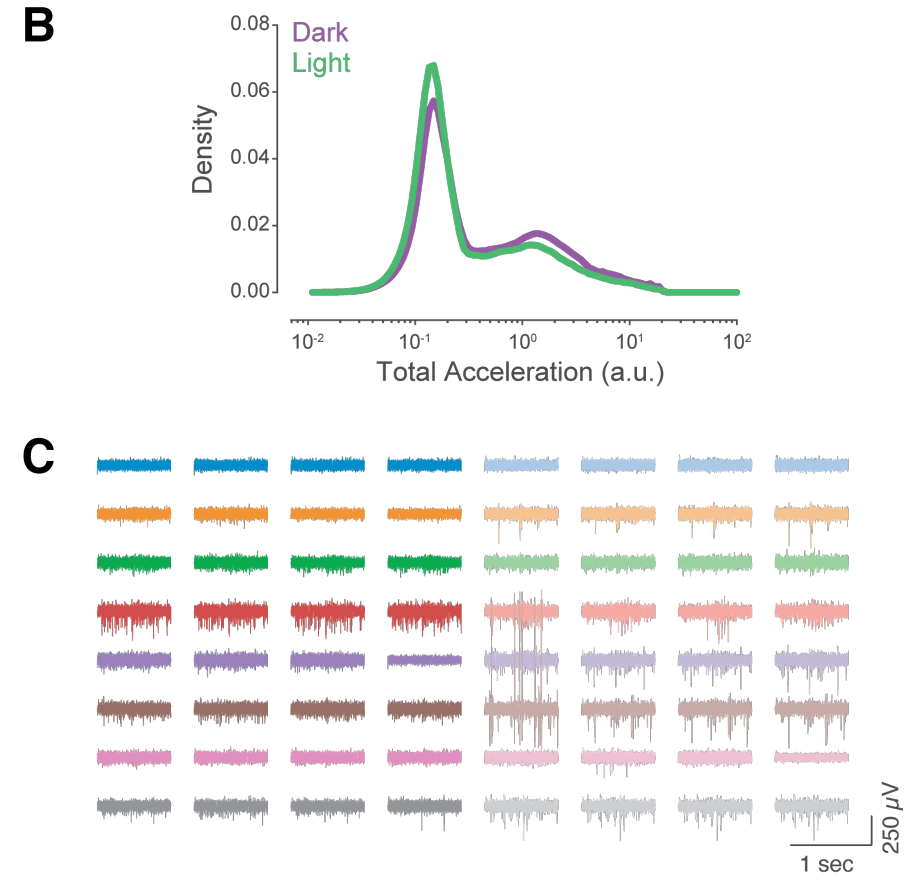

Figure S1: (Related to Figure 1). Details of Head Movement and V1 Neural Recordings. (a)

Example traces from a recording session showing yaw, roll, and pitch components of head direction over 100 minutes (top) or 100 seconds (bottom). (b) Mean histograms of total acceleration, defined as the norm of the three linear components of acceleration, for sessions recorded in dark (purple) or light (green). (c) Example raw bandpass-filtered traces showing spiking activity from one V1 recording session, colored by tetrode. 
bioRxiv preprint doi: https://doi.org/10.1101/2020.09.04.283762; this version posted September 5, 2020. The copyright holder for this preprint (which was not certified by peer review) is the author/funder, who has granted bioRxiv a license to display the preprint in perpetuity. It is made available under aCC-BY-NC-ND 4.0 International license.

A

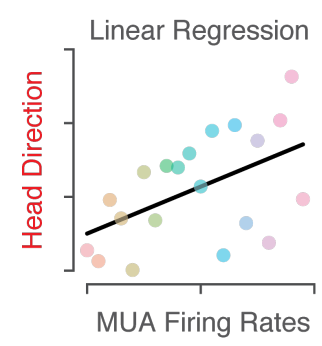

C

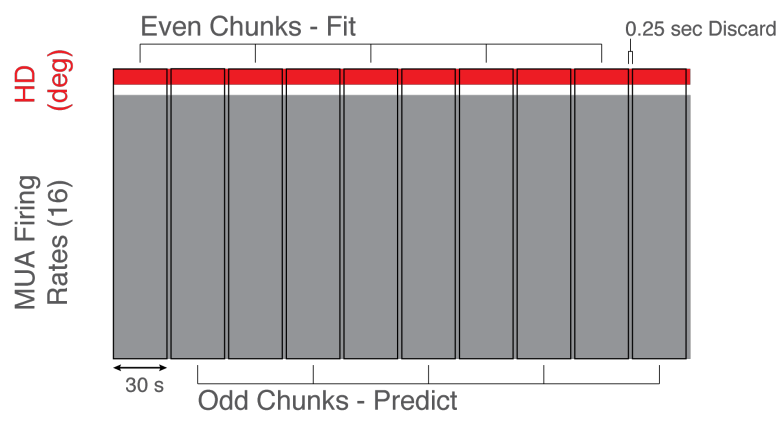

B

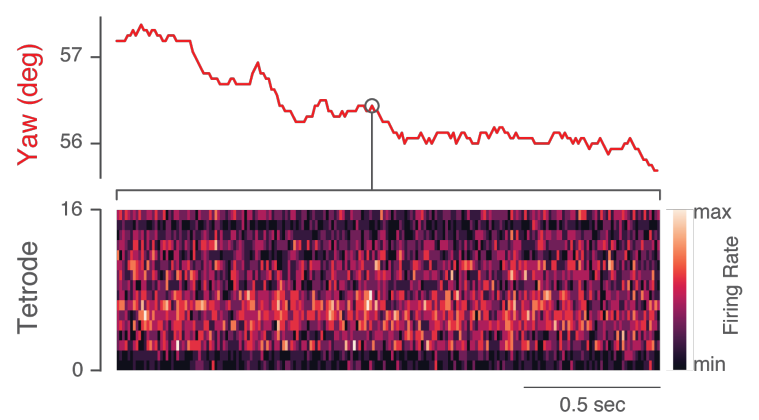

Figure S2: (Related to Figure 1). Head Direction Decoding Approach and Decoding in M2-Lesioned Animals. (a) Multiunit activity (MUA) vectors from the 16 tetrodes were used to predict yaw, roll, or pitch components of 3D HD separately, using ridge regression. (b) A vectorized MUA window ranging from $10 \mathrm{~ms}$. to 2 sec. was used to predict any given HD time-point. (c) Sessions were divided into 30-second even and odd chunks, with the former used for fitting the models and the latter for testing. The chunks were separated by 0.25 -second discarded gaps to ensure that model performance was not due to continuities in the HD or MUA signals. (d) Excitotoxic lesions to secondary motor cortex (M2) were performed bilaterally using ibotenic acid. Left: horizontal view of overlaid lesion ROIs from $n=4$ rats. Right: Ridge regression decoding results for non-lesioned $(n=5)$ and lesioned $(n=4)$ rats. Each dot represents one 2-hour session; outlines are violin plots. Yaw, dark: Non-lesioned: $r_{\text {circ }}=0.31 \pm 0.02$; Lesioned: $0.33 \pm 0.01$ (mean $\pm \mathrm{SEM}$ ). MWU test $p=0.4$. Yaw, light: Non-lesioned: $r_{\text {circ }}=0.30 \pm 0.02$; Lesioned: $0.31 \pm 0.01$. MWU test $p=0.31$. Roll, dark: Non-lesioned: $r=0.41 \pm 0.02$; Lesioned: $0.39 \pm 0.02$. MWU test $p=0.26$. Roll, light: Non-lesioned: $r=0.42 \pm 0.01$; Lesioned: $0.39 \pm 0.02$. MWU test $p=0.14$. Pitch, dark: Non-lesioned: $r=0.46 \pm 0.02$; Lesioned: $0.36 \pm 0.01$. MWU test $p=1.2 \times 10^{-5}$. Pitch, light: Non-lesioned: $r=0.44 \pm 0.02$; Lesioned: $0.36 \pm 0.02$. MWU test $p=7.5 \times 10^{-4}$. 
bioRxiv preprint doi: https://doi.org/10.1101/2020.09.04.283762; this version posted September 5, 2020. The copyright holder for this preprint (which was not certified by peer review) is the author/funder, who has granted bioRxiv a license to display the preprint in perpetuity. It is made available under aCC-BY-NC-ND 4.0 International license.

A

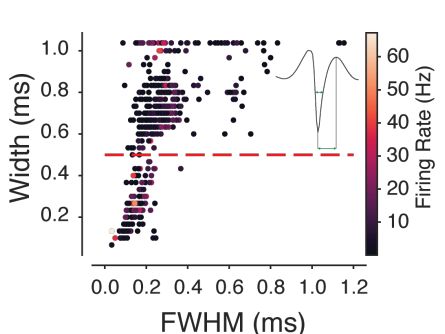

B

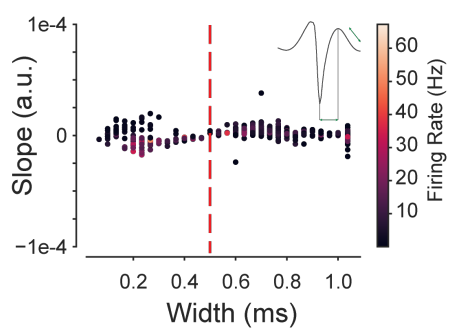

C

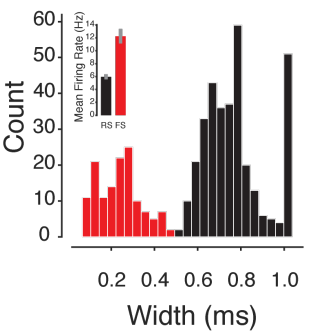

D

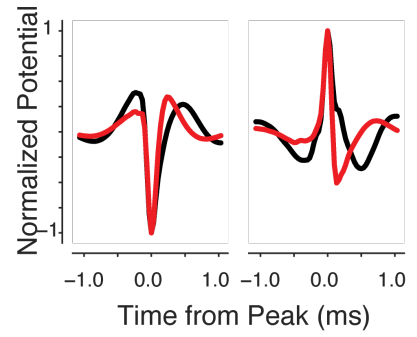

Figure S3: (Related to Figure 2). Single-Unit Waveform Classification. Units were classified into regular-spiking putative pyramidal units (RSUs) or fast-spiking putative inhibitory units (FSUs) based on calculations of spike width (trough to peak time), full-width-at-half-max (FWHM) time, or end-of-waveform slope. For classification, waveforms from the highest-amplitude channels within a tetrode were used. (a) Spike width vs. FWHM. Each dot represents a unit, colored by its mean firing rate. Inset: waveform illustrating width and FWHM calculation. Dotted line: classification boundary for FSUs (width $<0.5 \mathrm{~ms}$ ) and RSUs (width $>0.5 \mathrm{~ms}$ ). (b) Waveform slope vs. width. (c) Histogram of mean spike widths. Inset: Mean firing rates of RSUs and FSUs. Red: FSUs ( $n=61$ in dark; $n=50$ in light), Black: RSUs ( $n=142$ in dark; $n=112$ in light). (d) Mean waveforms of $n=365$ single units recorded in dark $(n=203)$ or light $(n=162)$ in $n=5$ rats across $n=10$ sessions, separated by negative-peaked (left) and positive-peaked (right) units. Color indicates fast-spiking units (FSUs, red) and regular-spiking units (RSUs, black) based on classification in c.

\section{A Example Waveforms Recorded in Dark and Light}

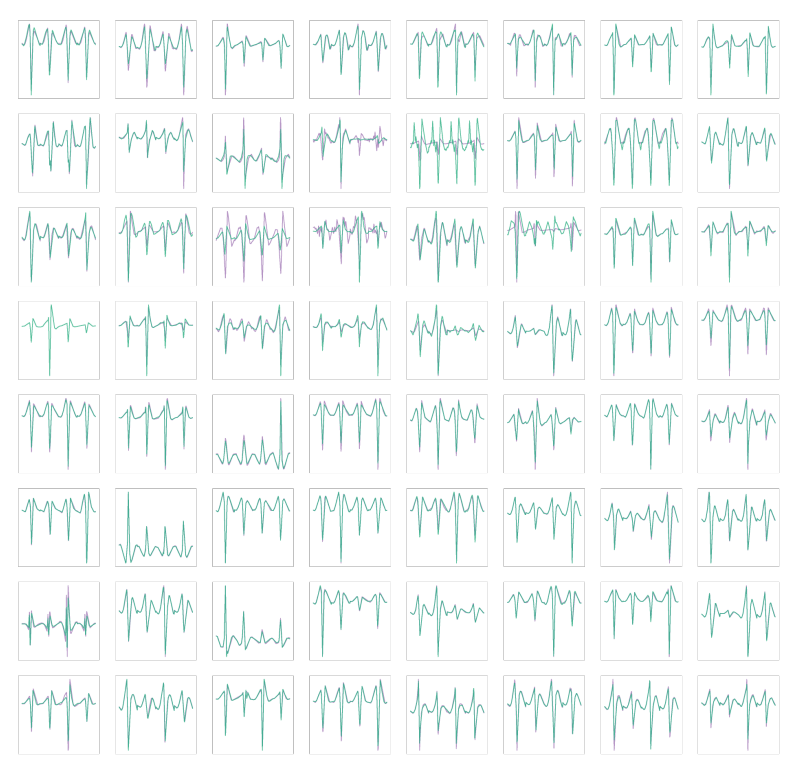

B

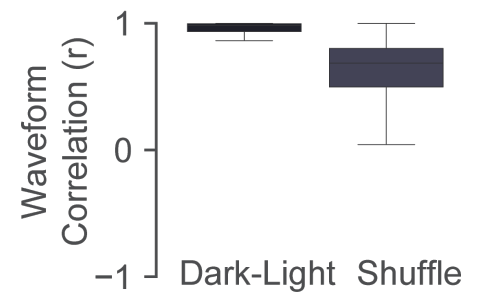

C

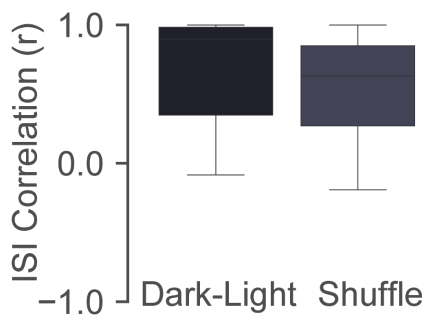

Figure S4: (Related to Figure 4). Tracking Single Units Across Dark and Light Sessions. (a) Example mean waveforms from tetrode channels for units sorted jointly across dark and light sessions. (b) Correlations between mean waveforms in dark and light $(r=0.93 \pm 0.12$, mean \pm SEM) were significantly higher than among $N=1000$ shuffled pairs $(r=0.55 \pm 0.41)$, $p=2.57 \times 10^{-58}$, MWU test. (c) Correlations between mean inter-spike-interval distributions for units recorded in dark and light $(r=0.70 \pm 0.34)$ were significantly higher than those among $N=1000$ shuffled pairs $(r=0.56 \pm 0.32), p=4.36 \times 10^{-09}$, MWU test. 